\title{
Built-in electric fields and valence band offsets in InN/GaN(0001) superlattices: First-principles investigations
}

\author{
C. C. Shieh, ${ }^{1}$ X. Y. Cui, ${ }^{1, a)}$ B. Delley, ${ }^{2}$ and C. Stampfl ${ }^{1}$ \\ ${ }^{1}$ School of Physics, The University of Sydney, Sydney, New South Wales 2006, Australia \\ ${ }^{2}$ Paul Scherrer Institut WHGA/123 CH-5232 Villigen PSI, Switzerland
}

(Received 14 December 2010; accepted 2 March 2011; published online 29 April 2011)

\begin{abstract}
Based on all-electron density functional theory calculations, we systematically investigate the built-in electric fields and valence band offsets in wurtzite $\mathrm{InN} / \mathrm{GaN}(0001)$ superlattices, where their correlations with biaxial strain, as well as the superlattice geometry, are determined. Both the built-in electric fields (several MV/cm) and the valence band offsets $(0.16-1.1 \mathrm{eV})$ are found to be strongly dependent on the superlattice geometry and strain growth conditions. Spontaneous polarization and strain-induced piezoelectric polarization are comparable in contribution to the macroscopic electric field. Relative to the fully relaxed superlattices, tensile (compressive) strain significantly weakens (strengthens) the magnitude of the electric field, and decreases (increases) the value of the valence band offset. The results will be valuable in relation to practical heterojunction-based device optimization and design. (C) 2011 American Institute of Physics. [doi:10.1063/1.3573499]
\end{abstract}

\section{INTRODUCTION}

The group III nitrides and their alloy systems represent a family of materials, with their room temperature bandgaps ranging from $0.64 \mathrm{eV}(\mathrm{InN}), 3.43 \mathrm{eV}(\mathrm{GaN})$, to $6.14 \mathrm{eV}$ (AlN), widely used in optoelectronic devices active at wavelengths from the red to the ultraviolet. ${ }^{1}$ Among the group III nitrides, InN has been recently drawing particular attention due to its superior electrical and optical properties (small effective mass, small bandgap, high electron drift velocity and high mobility), which make it promising for applications in high-frequency/high-speed/high-power heterojunction field-effect transistors (HFETs). ${ }^{1,2}$

Wurtzite (wz) group III nitrides are pyroelectric, in which large spontaneous polarizations exist along the $c-$ axis (polar axis); furthermore, depending on the degree of lattice mismatch, the piezoelectric effect also contributes to the polarization fields to a certain extent. The macroscopic polarization fields manifest themselves as bound interface charges at the heterojunction and induce the "built-in electric field," which is a vital parameter in determining bandgaps, band structure and other electronic properties, ${ }^{2}$ and has usually been expected to be a constant with respect to the superlattice (SL) geometry and strain conditions. ${ }^{3}$ However, we recently demonstrated that the magnitude of built-in electric fields in GaN/ AIN(0001) SLs can be tuned by control of the biaxial strain as well as by the thickness of both the well and barrier regions. ${ }^{4}$ This provides more flexibility in bandgap engineering. ${ }^{4,5}$ Furthermore, for the InN/GaN SLs, the contribution of the piezoelectric polarizations is expected to play a more significant role than that in the GaN/AlN system [which is only $5-10 \%$ (Ref. 4)], due to the much larger in-plane lattice mismatch between InN and $\mathrm{GaN}$ than that between $\mathrm{GaN}$ and $\mathrm{AlN} .^{3,6}$ Indeed, research is focused only on (In,Ga)N/GaN or similar alloyed systems ${ }^{7}$ because of the difficulty to fabricate pure

${ }^{\text {a)} E l e c t r o n i c ~ m a i l: ~ c a r l . c u i @ s y d n e y . e d u . a u . ~}$
InN/GaN multiple quantum well (MQW) systems. ${ }^{6}$ Recent advance in epitaxial growth technology has however eliminated this difficulty; several groups ${ }^{3,8-10}$ have grown InN films on GaN and AlN substrates by conventional or plasmaassisted molecular beam epitaxy (PA-MBE). There are very few measurements of the strength of the built-in electric fields in InN/GaN MQW systems and the reported values show a wide disparity. Via plotting electron drift velocity investigated by Raman spectroscopy as a function of the strength of the electric fields, Liang et al. ${ }^{9}$ reported a value of $0.075 \mathrm{MV} / \mathrm{cm}$ deduced from a wz $\operatorname{InN}(0001)$ film of thickness around 7.5 $\mu \mathrm{m}$ grown on wz GaN(0001) layers. Lai et al. ${ }^{11}$ investigated the alloyed $\operatorname{In}_{0.23} \mathrm{Ga}_{0.77} \mathrm{~N} / \mathrm{GaN}(0001)$ system by electron transmission spectroscopy, where the magnitude of the electric fields resulting only from the piezoelectric effect was determined to be $1.7-1.9 \mathrm{MV} / \mathrm{cm}$. This value is expected to be higher in the InN/GaN system, because of the larger contribution of both spontaneous and piezoelectric components, as found in GaN/AIN systems. ${ }^{12}$ Considering only the piezoelectric contribution, a theoretical prediction made by Martin et $a l .{ }^{13}$ via the piezoelectric coefficients gives values of $5.5 \mathrm{MV} /$ $\mathrm{cm}$ and 2.1 MV/cm for a wz $\mathrm{InN}(0001)$ film grown on a $\mathrm{GaN}$ substrate and for a wz GaN(0001) film grown on an InN substrate, respectively. This derivation indicates that strain plays an important role in affecting the built-in electric fields. To our knowledge, there is no theoretical or experimental investigation of the dependence of the built-in electric fields on the geometry of InN/GaN(0001) SLs.

The valence band offset (VBO) is another fundamental quantity in affecting the electronic and transport properties of heterostructures and consequently is vital to the design of InNbased optoelectronic devices. ${ }^{1}$ There has been a considerable effort from both experimental and theoretical sides, ${ }^{3,10,13-20}$ to determine this quantity. However, there is no consensus on the $\mathrm{VBO}$ of InN/GaN, with the calculated values ranging from 0.3 $\mathrm{eV}$ to $1.27 \mathrm{eV}$, and the experimental data from $0.5 \mathrm{eV}$ to 1.1 $\mathrm{eV}$ (see Ref. 1). The value of $1.05 \pm 0.25 \mathrm{eV}$ obtained by 
Martin et $a l .{ }^{13}$ by using X-ray photoemission spectroscopy (XPS) over a decade ago has endeavored to eliminate the piezoelectric effect by introducing a correction term; as pointed out by King et al., ${ }^{10}$ the correction term is, however, based on a false assumption that the piezoelectric effect always acts to decrease the VBO value, which does not consider the fact that the piezoelectric coefficients have different signs when $\mathrm{InN}$ films are coherently strained on $\mathrm{GaN}$ (compressive strain) or when GaN films are coherently strained on $\mathrm{InN}$ (tensile strain). ${ }^{21}$ Also using XPS, King et al. ${ }^{10}$ recently reported a value of $0.58 \pm 0.08 \mathrm{eV}$ for the same system. There are also a few first-principles studies of the VBO. Wei and Zunger ${ }^{14}$ determined the VBO of unstrained (i.e., atomically relaxed) InN/GaN(0001) SLs to be $0.48 \pm 0.1$ or $0.26 \pm 0.1 \mathrm{eV}$ in the wZ or zinc-blende $(\mathrm{zb})$ structure by density functional theory (DFT) calculations. In the same paper, it was also suggested that the VBO for the $\mathrm{zb}$ structure is lower than that for the corresponding wz structure, which was believed to be due to the difference in the strain relaxation at the interface. Recently, a large natural VBO value of $1.11 \mathrm{eV}$ was reported for $\mathrm{zb}-\mathrm{InN} /$ GaN heterostructure. ${ }^{19}$ Van de Walle and Neugebauer ${ }^{22}$ also performed DFT calculations to determine the VBO for the $\mathrm{zb}$ InN/GaN(110) heterojunction to be $0.3 \pm 0.1 \mathrm{eV}$. Recently, using a hybrid exchange-correlation functional, Moses and Van de Walle reported the $\mathrm{InN} / \mathrm{GaN}(0001) \mathrm{VBO}$ value of 0.62 eV (Ref. 20). All the theoretical studies employed a model of the SL as having the same number atomic layers for the $\mathrm{InN}$ and $\mathrm{GaN}$ regions. To our knowledge, there are no systematic experimental or first-principles studies of the VBOs for $\mathrm{InN} /$ $\mathrm{GaN}$ systems for differing thickness of $\mathrm{GaN}$ and $\mathrm{InN}$ regions, nor as a function of strain.

This paper, therefore, will provide an extensive investigation of the built-in electric fields and VBOs in wz InN/ GaN(0001) SL systems through DFT calculations. We find that depending on the strain involved, piezoelectric polarization is comparable with spontaneous polarization in $\mathrm{InN} /$ $\mathrm{GaN}(0001)$ SLs. More importantly, in sharp contrast to common understanding, our results demonstrate that both the built-in electric fields and VBOs are not constants as widely assumed or rather, both depend markedly on the SL geometry and strain conditions. Thus, our theoretical predictions represent a valuable guide to the practical engineering of such $\mathrm{InN} / \mathrm{GaN}$ based heterojunction devices.

\section{METHOD}

\section{A. Computational details}

We perform all-electron density functional theory (DFT) calculations using the generalized gradient approximation $^{23}$ (GGA) for the exchange correlation functional as implemented in the $\mathrm{DMol}^{3}$ code. ${ }^{24}$ The wave functions are expanded in terms of a double-numerical quality localized basis set with a real-space cutoff of 9 Bohr. Using periodic SLs, we simulate the wz InN/GaN(0001) MQW with Ga (In) polarity which are composed of a large number of alternating wz InN (well) and GaN (barrier) layers. For convenience of notation, we denote the number of well layers as $m$ and that of barrier layers as $n$. A general InN/GaN SL can then be described as $m+n$. Figure 1 displays an example of a SL
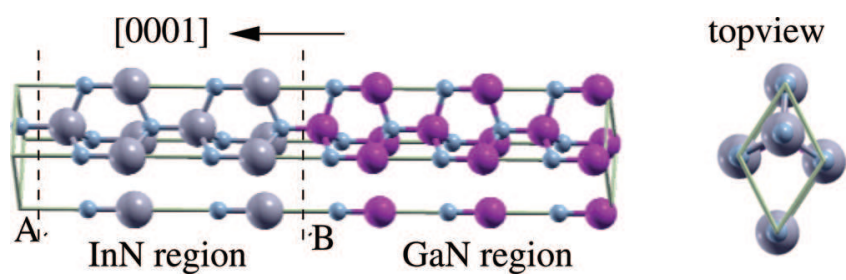

FIG. 1. (Color online) Illustration of the geometry of a $4+6 \mathrm{wz} \mathrm{InN} /$ $\mathrm{GaN}(0001)$ superlattice. The two vertical dashed lines represent the two types of interfaces $A$ and $B$. Small (green) spheres indicate $N$ atoms, and large (gray) and medium (pink) spheres indicate In and $\mathrm{Ga}$ atoms, respectively.

structure, namely a $4+6 \mathrm{wz} \operatorname{InN} / \mathrm{GaN}(0001) \mathrm{SL}$ with Ga (In) polarity, which contains a type A interface (defined to be the interface from well to barrier along the [0001] direction) and a type B interface (defined to be the interface from barrier to well along the [0001] direction). The Brillouinzone integrations are performed using a $10 \times 10 \times 4 \mathrm{k}$-point grid for the $6+6$ structure. For other SLs, the same/similar sampling of reciprocal space is used.

Three approaches of relaxation are applied to mimic different strain conditions while finding the atomic geometry which minimizes the total energy. The first approach involves "full relaxation," where all the lattice constants and internal parameters are free to vary until the system reaches a "strainfree" (free-standing) configuration. ${ }^{25}$ The second and third is to investigate the effect of strain; here we relax only $c$ and all the internal atomic coordinates, but fix the in-plane lattice constant $a$ to be either that of (i) InN, in which case the system experiences tensile strain due to the larger lattice constant of $\mathrm{InN}$, or conversely, that of (ii) $\mathrm{GaN}$, in which case the system experiences compressive strain. In all three approaches, we impose "pseudomorphic growth," assuming that the in-plane lattice constants in the $\mathrm{InN}$ and $\mathrm{GaN}$ regions match completely, thus no lattice defects are generated.

\section{B. Strength of electric fields}

The presence of a built-in electric field can be demonstrated by using the core-levels as reference energies to determine the relative alignment of the valence band edges. ${ }^{26,27}$ Here, we use the $\mathrm{N}-1 s$ orbital binding energy, $E_{b}^{C L}$, in different layers, for various sized SLs. For an example, Fig. 2 shows a plot of the core-level eigenvalue $E_{b}^{C L}$ with respect to the distance in the [0001] direction (z-coordinate) of a $8+8$ strain-free system, where the core-level eigenvalues are tilted by the electric fields, resulting in a "V-shaped" profile. The absolute value of the slope of the line of best fit in each region corresponds to the magnitude of the built-in electric field in the corresponding region, ${ }^{27}$ namely

$$
|E|=\left|\Delta E_{b}^{C L} / \Delta z\right|
$$

The V-shaped profile implies that the built-in electric fields point from the type B interface to the type A interface in both well and barrier regions.

\section{Valence band offset}

We calculate the VBO according to the formula: ${ }^{28}$ 


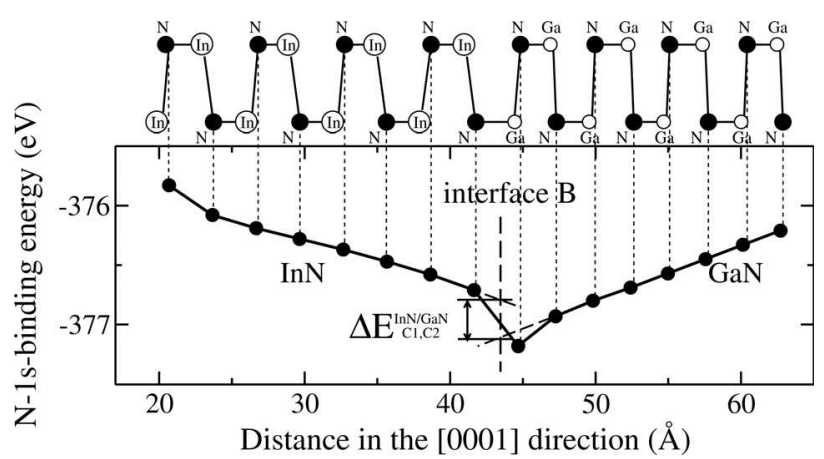

FIG. 2. Illustration of the calculation of the interface term $\Delta E_{C 1, C 2}^{\mathrm{GaN} / \mathrm{InN}}$ by linear extrapolation of the gradients in each region of a $8+8$ strain free system. The position of the N-1s core-levels (circles) is plotted vs distance in the superlattices, where the lines are to guide the eyes. The corresponding atomic structure is depicted above.

$$
\Delta E_{v}(\mathrm{GaN} / \mathrm{InN})=\left(\Delta E_{v 2, C 2}^{\mathrm{InN}}-\Delta E_{\mathrm{v} 1, \mathrm{C} 1}^{\mathrm{GaN}}\right)+\Delta E_{C 1, C 2}^{\mathrm{GaN} / \mathrm{InN}}
$$

where $\Delta E_{v}(\mathrm{GaN} / \mathrm{InN})$ is the $\mathrm{VBO}, \Delta E_{v 1, C 1}^{\mathrm{GaN}}$ (and similarly for $\left.\Delta E_{v 2, C 2}^{\mathrm{InN}}\right)$ is the binding energy difference between the core-level $(C 1)$ and the valence band maximum (VBM, $v 1$ ) for pure $\mathrm{GaN}$ (and similarly for pure $\mathrm{InN}$ ). Here we use the $\mathrm{N}-1 s$ orbital binding energy as the core-level eigenvalue. $\left(\Delta E_{v 2, C 2}^{\mathrm{InN}}-\Delta E_{v 1, C 1}^{\mathrm{GaN}}\right)$ is the so called "bulk term." For a given $\mathrm{SL}$, the bulk term is calculated for the bulk material having the in-plane lattice constant $a$ being that of the corresponding $\mathrm{SL}$ and the $c$ lattice constant free for relaxation. $\Delta E_{\mathrm{C} 1, \mathrm{C} 2}^{\mathrm{GaN} / \mathrm{InN}}$, or the so called "interface term", is defined to be:

$$
\Delta E_{\mathrm{C} 1, \mathrm{C} 2}^{\mathrm{GaN} / \mathrm{InN}}=\mathrm{E}_{\mathrm{b}}^{\mathrm{CL}, \mathrm{InN}}-\mathrm{E}_{\mathrm{b}}^{\mathrm{CL}, \mathrm{GaN}}
$$

where $E_{b}^{C L, \mathrm{InN}}$ and $E_{b}^{C L, \mathrm{GaN}}$ are the binding energies of the core-level $\mathrm{N}-1 s$ orbital in the $\mathrm{InN}$ and $\mathrm{GaN}$ regions, respectively. ${ }^{28}$ In the present SL systems, the V-shaped profile of the core-level eigenvalue, caused by the quantum confined Stark effect, ${ }^{29}$ makes it hard to define the difference in $E_{b}^{C L}$ at the heterojunction, hence the interface term. We follow the method used by Picozzi et al., ${ }^{27}$ where the interface term is calculated by finding the difference of the core-level eigenvalues at the interface plane obtained by linear extrapolation, as illustrated by Fig. 2, where an $8+8$ SL is taken as an example. In this paper, we will use the conventional interface plane for the extrapolation procedure, which is at the midpoint of the heterojunction. ${ }^{27}$ Note that the reversing of the order of the heterojunction (i.e., InN/GaN) simply results in VBOs with an opposite sign. We choose GaN/InN to correspond with the common convention that the compound on the right-hand side has a higher value of the VBM. ${ }^{14}$

\section{RESULTS}

\section{A. Bulk properties}

Firstly, we consider the basic properties of the parent compounds- wz bulk InN and GaN. The lattice constants, internal parameters, bandgaps, heat of formation and cohesive energies of strain-free bulk InN and GaN are given in Table I. For crystalline structural parameters, our results compare well
TABLE I. Lattice constants $a, c$ and $c / a$, internal parameter $u$, bandgap $E_{g}$, heat of formation $\Delta H_{f}$ and cohesive energy $E_{c}$.

\begin{tabular}{llllllll}
\hline \hline Method & $a(\AA)$ & $c(\AA)$ & $c / a$ & $u$ & $E_{g}(\mathrm{eV})$ & $\Delta H_{f}(\mathrm{eV})$ & $E_{c}(\mathrm{eV})$ \\
\hline $\begin{array}{l}\text { InN bulk } \\
\text { Present }\end{array}$ & 3.583 & 5.802 & 1.619 & 0.379 & -0.23 & -0.063 & 7.289 \\
GGA & & & & & & & \\
GGA $^{\mathrm{a}}$ & 3.614 & 5.884 & 1.628 & 0.377 & -0.37 & & 6.872 \\
GGA $^{\mathrm{b}}$ & 3.5848 & 5.8002 & 1.6180 & 0.3793 & & -0.125 & 7.695 \\
Exp. $^{\mathrm{c}}$ & 3.538 & 5.703 & 1.6119 & & & $-0.21^{\mathrm{d}}$ & $7.970^{\mathrm{a}}$ \\
GaN bulk & & & & & & \\
Present & 3.229 & 5.259 & 1.629 & 0.377 & 1.81 & -0.873 & 8.785 \\
GGA & & & & & & \\
GGA $^{\mathrm{a}}$ & 3.245 & 5.296 & 1.632 & 0.3762 & 1.45 & & 8.265 \\
GGA $^{\mathrm{b}}$ & 3.1986 & 5.2262 & 1.6339 & 0.3762 & & -1.118 & 9.265 \\
Exp. $^{\mathrm{c}}$ & 3.1890 & 5.1863 & 1.6263 & 0.377 & & $-1.08^{\mathrm{d}}$ & $9.058^{\mathrm{a}}$ \\
\hline \hline
\end{tabular}

${ }^{\mathrm{a}}$ Reference 30. Pseudopotential plane-wave method.

${ }^{\mathrm{b}}$ Reference 31 . Pseudopotential plane-wave method.

${ }^{\mathrm{c}}$ Reference 32.

${ }^{\mathrm{d}}$ Reference 33 .

with the recommended data sorted by Vurgaftman and Meyer, ${ }^{6}$ other DFT-GGA calculations ${ }^{30,31}$ and experimental values. ${ }^{32}$ The bandgaps of both bulk $\mathrm{InN}$ and $\mathrm{GaN}$ are underestimated, which is typical of GGA. The lattice mismatch between wz InN and $\mathrm{GaN}$ is calculated to be $9.88 \%$ in $a$ and $9.36 \%$ in $c$, which is comparable to the suggested experimental values of $10.04 \%$ and $9.08 \%$, respectively. ${ }^{6}$ For both bulk $\mathrm{InN}$ and $\mathrm{GaN}$, the internal parameter $u$, which is sensitive to polarization fields, is larger than the ideal value for the wz structure $(3 / 8=0.375)$, where a larger deviation from this value corresponds to a larger spontaneous polarization parameter (InN: $\left.-0.042 \mathrm{C} \mathrm{m}^{-2}, \mathrm{GaN}:-0.034 \mathrm{C} \mathrm{m}^{-2}\right){ }^{1}$

\section{B. Strain-free $m=n$ systems}

Figure 3 displays the N-1s binding energy of strain-free SLs with number of layers varying from $2+2$ to $10+10$. As the number of layers increases, the N-1s binding energy is shifted down with the $\mathrm{V}$-shaped profile remaining. The magnitude of the electric fields and lattice constants and VBOs of these systems are summarized in Table II. The $2+2$ system is excluded because of the very large, but less

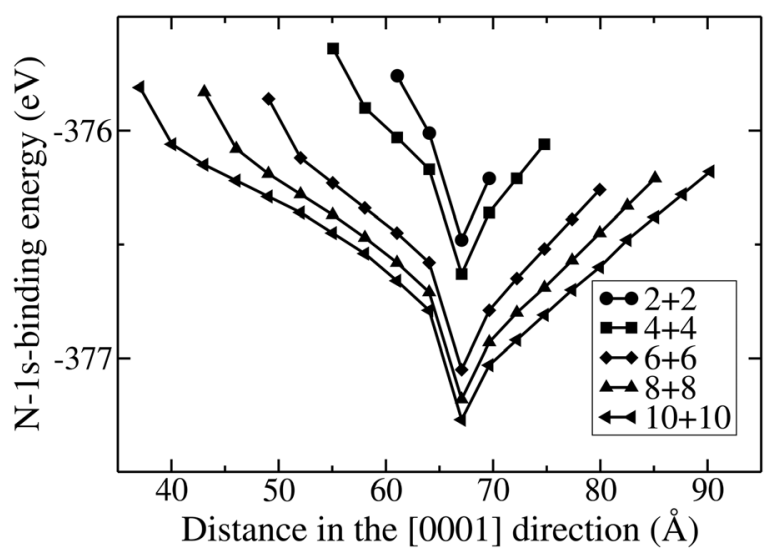

FIG. 3. The N-1s binding energy of strain-free wz InN/GaN(0001) SLs as a function of $z$ (distance in the [0001] direction), for the number of layers varying from $2+2$ to $10+10$. 
TABLE II. The magnitude of the electric fields $|E|$, lattice constants, bulk terms, interface terms and VBOs of strain-free wz InN/GaN(0001) SLs, with the number of layers varying from $2+2$ to $10+10$.

\begin{tabular}{llllll}
\hline \hline$m=n$ Systems & $2+2$ & $4+4$ & $6+6$ & $8+8$ & $10+10$ \\
\hline$a(\AA)$ & 3.405 & 3.406 & 3.402 & 3.402 & 3.402 \\
$c / n(\AA)$ & 5.565 & 5.563 & 5.574 & 5.574 & 5.574 \\
$|E|_{\text {InN }}(\mathrm{MV} / \mathrm{cm})$ & & 4.51 & 3.80 & 3.41 & 2.92 \\
$|E|_{\mathrm{GaN}}(\mathrm{MV} / \mathrm{cm})$ & & 5.83 & 5.12 & 4.63 & 4.14 \\
bulk term $(\mathrm{eV})$ & 0.099 & 0.102 & 0.107 & 0.107 \\
interface term $(\mathrm{eV})$ & & 0.361 & 0.366 & 0.375 & 0.408 \\
VBO $(\mathrm{eV})$ & & 0.460 & 0.468 & 0.482 & 0.515 \\
\hline
\end{tabular}

reliable value of electric field due to the difficulty of defining well (barrier) regions and the interface region. As expected, the in-plane lattice constants of these systems are between that of $\mathrm{InN}$ and $\mathrm{GaN}$. The magnitude of the electric fields is around a few $\mathrm{MV} / \mathrm{cm}$, which is considerable, and decreases with increasing number of both layers, with the values in the barrier region always higher than those in the well region due to the thinner thickness of the barrier region $\left(c_{\mathrm{GaN}}\right.$ is smaller than $c_{\text {InN }}$ ). This result is different from the $\mathrm{GaN} /$ AlN(0001) heterojunction, where the magnitude of the electric fields is essentially a constant of $5.1 \pm 0.3 \mathrm{MV} / \mathrm{cm}^{4}$ for varying $n$ in the $n+n$ superlattices.

Summaries of the calculated VBOs and the constituent components, namely the bulk term and the interface term, are listed in Table II. The VBOs increase with an increasing number of both layers, ranging from $0.460 \mathrm{eV}$ to $0.515 \mathrm{eV}$, where the changes mainly result from the interface terms, due to the shape of the "core-level shift." Also employing DFT calculations, Wei and Zunger ${ }^{14}$ determined the magnitude of the VBO for a (0001) heterojunction of $\mathrm{GaN} / \mathrm{InN}$ having the in-plane lattice constant equal to the average of that of $\mathrm{GaN}$ and $\mathrm{InN}$ to be $0.48 \pm 0.1 \mathrm{eV}$, which lies well between the range we obtain for the fully optimized structures.

\section{Strain-free $\boldsymbol{m} \neq \boldsymbol{n}$ systems}

To focus on the effect of the SL geometry, we decrease the number of either well or barrier layers, resulting in $m \neq n$ systems. Figure 4 displays the $\mathrm{N}-1 s$ binding energy of $6+8,8+8$ and $8+6$ strain-free systems with respect to $z$ (the distance in the [0001] direction). Comparing with the $8+8$ case, when the number of well layers is reduced $(6+8)$, the core-level eigenvalues and the magnitude of the electric field increase in the well region, while those in the barrier region are barely affected. On the other hand, when the number of barrier layers is reduced $(8+6)$, core-level eigenvalues in both regions are shifted up, but resulting in a weaker electric field in the well region and an almost identical electric field in the barrier region. The trend repeats itself for cases around a $6+6$ system $(4+6,6+6$ and $6+4)$. Results for the magnitude of the electric fields and lattice constants for $m \neq n$ SLs are summarized in Table III, with their values compared with the corresponding $m=n$ systems. The values of the bulk and interface terms are also listed.

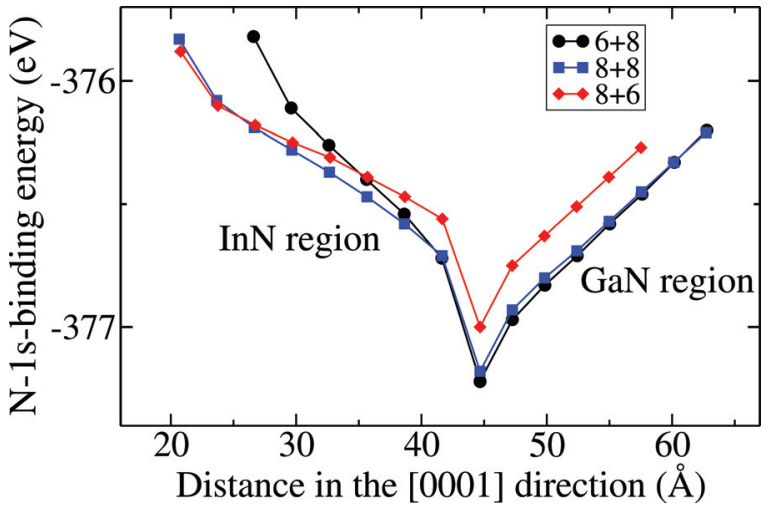

FIG. 4. (Color online) The $\mathrm{N}-1 s$ binding energy of strain-free $6+8,8+8$ and $8+6 \mathrm{wz} \mathrm{InN} / \mathrm{GaN}(0001) \mathrm{SLs}$ as a function of $z$, distance in the [0001] direction.

Both lattice constants $a$ and $c / n$ decrease (increase) with decreasing number of well (barrier) layers, which is expected from the larger lattice constants of the well compound ( $\mathrm{InN})$. The change in the magnitude of the electric fields in the well region due to decrease in the number of well or barrier layers is much more significant than that in the barrier region.

From Table III, it can be seen that there are considerable changes in $a$ between the $4+6 / 6+6 / 6+4$ (or $6+8 / 8+8$ / $8+6)$ SL systems, with a corresponding decrease in the bulk term with increasing value of $a$. The interface terms also decrease as $a$ increases, resulting in VBOs ranging from $0.371(a=3.442 \AA)$ to $0.563 \mathrm{eV}(a=3.360 \AA)$. There is a similar trend for the $6+8 / 8+8 / 8+6$ superlattice systems. This reveals the relationship and influence of the SL geometry (including the value of $a$ ) on the interface and bulk terms, and the VBOs. King et al. ${ }^{10}$ determined the magnitude of the VBO for an unstrained wz $\operatorname{InN}(0001)$ film with thickness $5 \pm 1 \mathrm{~nm}$ grown on a thicker GaN template via XPS, where the strain relaxes within the first few monolayers, to be $0.58 \pm 0.08 \mathrm{eV}$. This value is comparable, though somewhat larger than, the magnitude of all our unstrained results as shown in Table III for the various SLs. We note that the experimental results, however, correspond to a much thicker well and barrier region than our SLs. As seen from Table II, there is a trend of increasing VBO with increasing number of layers, e.g., for a $4+4 \mathrm{SL}$, it is $0.460 \mathrm{eV}$ and for a $10+10$ $\mathrm{Sl}$, the value is $0.515 \mathrm{eV}$.

TABLE III. The magnitude of the electric fields $|E|$, lattice constants and VBOs of strain-free wz InN/GaN(0001) $4+6,6+4,6+8$ and $8+6$ SLs, compared with the corresponding $m=n$ systems.

\begin{tabular}{lllllll}
\hline \hline$m+n$ Systems & $4+6$ & $6+6$ & $6+4$ & $6+8$ & $8+8$ & $8+6$ \\
\hline$a(\AA)$ & 3.360 & 3.402 & 3.442 & 3.372 & 3.402 & 3.431 \\
$c / n(\AA)$ & 5.516 & 5.574 & 5.627 & 5.627 & 5.574 & 5.612 \\
$|E|_{\text {InN }}(\mathrm{MV} / \mathrm{cm})$ & 6.31 & 3.80 & 2.32 & 4.99 & 3.41 & 2.51 \\
$|E|_{\text {InN }}(\mathrm{MV} / \mathrm{cm})$ & 5.37 & 5.12 & 4.87 & 4.92 & 4.63 & 4.67 \\
bulk term $(\mathrm{eV})$ & 0.188 & 0.102 & 0.034 & 0.162 & 0.107 & 0.053 \\
interface term $(\mathrm{eV})$ & 0.375 & 0.366 & 0.337 & 0.384 & 0.375 & 0.354 \\
VBO $(\mathrm{eV})$ & 0.563 & 0.468 & 0.371 & 0.547 & 0.482 & 0.407 \\
\hline \hline
\end{tabular}


(a)

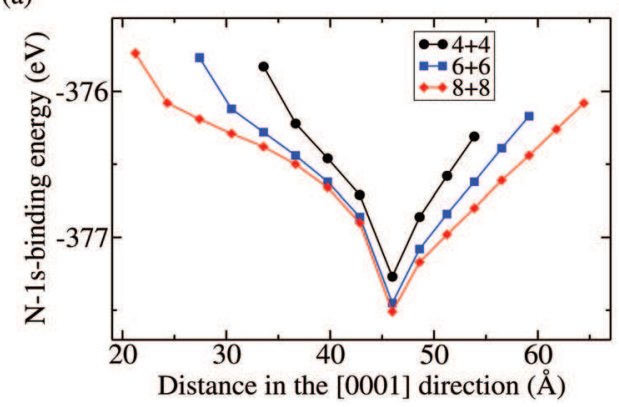

(b)

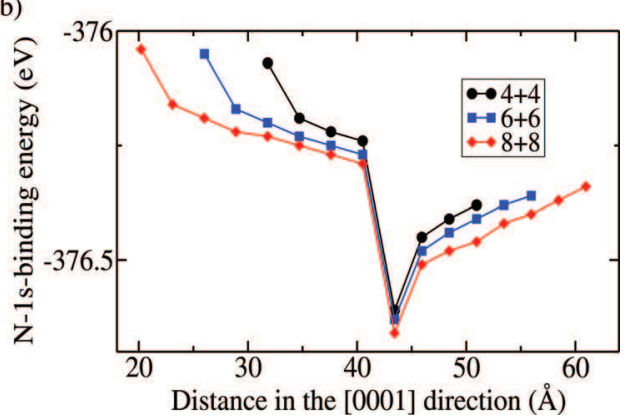

FIG. 5. (Color online) The N-1s binding energy of $4+4,6+6$ and $8+8$ wz InN/ $\mathrm{GaN}(0001)$ SLs under (a) compressive strain (in-plane lattice constant fixed at that of GaN "fix-GaN"), and (b) tensile strain (in-plane lattice constant fixed at that of InN "fix-InN") as a function of $z$, distance in the [0001] direction.

\section{Strained $\boldsymbol{m}=\boldsymbol{n}$ systems}

To investigate the effect of piezoelectric polarization, strain is applied to the InN/GaN superlattices. In particular, the in-plane lattice constant $a$ of bulk $\mathrm{InN}$ is larger than that of bulk GaN, corresponding to tensile (compressive) strain when the system is relaxed but with $a$ being fixed at that of bulk InN (GaN). Under strain, the V-shaped profile of the $\mathrm{N}$ $1 s$ core-level as a function of the number of layers as observed previously for the strain-free cases, remains, as shown in Fig. 5. Figure 6 shows a comparison for the $8+8$ SL under compressive and tensile strain with the corresponding strain-free system. In both well and barrier regions, the core-level eigenvalues are largely shifted up (down) by tensile (compressive) strain. Moreover, there are much flatter (steeper) linear regions corresponding to the much weaker (stronger) electric fields when the system is under tensile (compressive) strain. It can be seen that the magnitude of the electric fields is strongly affected by strain, e.g., for a $4+4$ $\mathrm{SL}$, the value in the well region varies from $0.86 \mathrm{MV} / \mathrm{cm}$ (fix-InN), $4.51 \mathrm{MV} / \mathrm{cm}$ (free), to $7.93 \mathrm{MV} / \mathrm{cm}$ (fix-GaN). This can be compared with analogous results for the GaN/ AlN system, where strain only contributes $5-10 \%$ to the electric field, indicating that the strain-induced piezoelectric polarizations are more dominant in wz InN/GaN SLs (Ref. 4). This is as expected from the large lattice mismatch between InN and GaN. Based on X-ray photoemission spectroscopy, Martin et al. ${ }^{13}$ calculated the magnitude of the

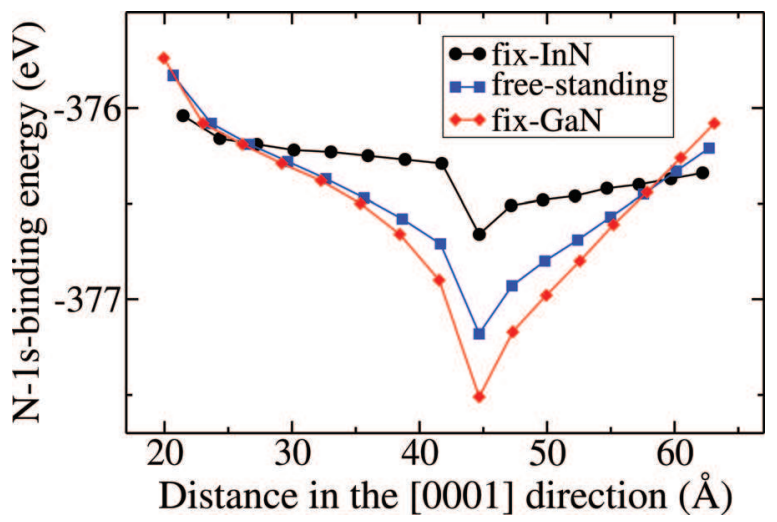

FIG. 6. (Color online) The N-1s binding energy of the $8+8 \mathrm{wz}$ InN/ $\mathrm{GaN}(0001)$ SLs under tensile (fix-InN), compressive (fix-GaN) and no strain (free-standing), as a function of $z$, distance in the [0001] direction. electric fields of an InN film grown on a GaN substrate and a GaN film grown on an InN substrate to be $5.5 \mathrm{MV} / \mathrm{cm}$ and 2.1 MV/cm, respectively. Though the strain condition was not stated clearly, we assume that the calculation was based on coherent growth, which corresponds to systems under compressive and tensile strain, respectively. The value 5.5 $\mathrm{MV} / \mathrm{cm}$ lies between our results for the $\mathrm{InN}$ region in $4+4$, $6+6$ and $8+8$ systems under compressive strain, with values of $7.93,5.89$ and $4.18 \mathrm{MV} / \mathrm{cm}$, respectively. The value $2.1 \mathrm{MV} / \mathrm{cm}$ is, however, higher than our results for the $\mathrm{GaN}$ region in $4+4,6+6$ and $8+8$ systems under tensile strain $(1.40,1.20$ and $1.13 \mathrm{MV} / \mathrm{cm})$ by over $0.7 \mathrm{MV} / \mathrm{cm}$.

The results for the VBOs are summarized in Table IV. It can be seen that strain also affects the VBO strongly, with the bulk and interface terms varying significantly according to different strain conditions, where both the interface terms and VBOs are lower (higher) when tensile (compressive) strain is applied. For example, for a $6+6 \mathrm{SL}$, the values of VBOs vary from $0.165 \mathrm{eV}$ (fix-InN), $0.468 \mathrm{eV}$ (free) and $1.068 \mathrm{eV}$ (fix-GaN). A similar trend holds for the case of $4+4$ and $8+8$ SLs. Note that though the bulk term also decreases with increasing $a$, it has a negative value when tensile strain is applied; furthermore, the deviation from the corresponding strain-free case for the bulk term is more significant for cases under compressive strain than tensile strain. Considering that applying tensile strain means fixing the $a$ of the SL to a larger value, the results here are still consistent with the previous ones, such that the VBO decreases with increasing $a$ (since both the interface and bulk terms do). The considerable effect of strain was also shown by Picozzi et al. ${ }^{27}$ in zb $3+3 \mathrm{GaSb} / \mathrm{InSb}(111)$ SLs.

\section{E. Strained $\boldsymbol{m} \neq \boldsymbol{n}$ systems}

Based on the aforementioned results, one will expect that the combination of SL geometry (that is, $m \neq n$ ) and strain will further enhance the flexibility of tuning the electric field and VBO of the InN/GaN SLs. To this end, we examine the effect of the SL geometry in strained systems. As the number of either well or barrier layers decreases, a similar but not identical trend to the corresponding $m \neq n$ strain-free case occurs: the decrease in the number of layers of a certain region increases only the core-level eigenvalues and the magnitude of the electric fields in the particular region, while that of the other region stays almost 
TABLE IV. The magnitude of the electric fields $|E|$, lattice constants and VBOs of wz InN/GaN(0001) $4+4,6+6$ and $8+8$ SLs under tensile and compressive strain, compared with the corresponding strain-free systems.

\begin{tabular}{|c|c|c|c|c|c|c|c|c|c|}
\hline \multirow{2}{*}{$\begin{array}{l}m=n \text { Systems } \\
\text { Relaxation }\end{array}$} & \multicolumn{3}{|c|}{$4+4$} & \multicolumn{3}{|c|}{$6+6$} & \multicolumn{3}{|c|}{$8+8$} \\
\hline & Fix-InN & Free & Fix-GaN & Fix-InN & Free & Fix-GaN & Fix-InN & Free & Fix-GaN \\
\hline$a(\AA)$ & 3.583 & 3.406 & 3.228 & 3.583 & 3.402 & 3.228 & 3.583 & 3.402 & 3.228 \\
\hline$c / n(\AA)$ & 5.405 & 5.563 & 5.723 & 5.405 & 5.574 & 5.723 & 5.405 & 5.574 & 5.723 \\
\hline$|E|_{\mathrm{InN}}(\mathrm{MV} / \mathrm{cm})$ & 0.86 & 4.51 & 7.93 & 0.86 & 3.80 & 5.89 & 0.71 & 3.41 & 4.18 \\
\hline$|E|_{\mathrm{GaN}}(\mathrm{MV} / \mathrm{cm})$ & 1.40 & 5.83 & 10.43 & 1.20 & 5.12 & 8.61 & 1.13 & 4.63 & 6.87 \\
\hline bulk term $(\mathrm{eV})$ & -0.070 & 0.099 & 0.553 & -0.070 & 0.102 & 0.553 & -0.070 & 0.107 & 0.553 \\
\hline interface term $(\mathrm{eV})$ & 0.250 & 0.361 & 0.464 & 0.235 & 0.366 & 0.515 & 0.254 & 0.375 & 0.571 \\
\hline $\mathrm{VBO}(\mathrm{eV})$ & 0.180 & 0.460 & 1.017 & 0.165 & 0.468 & 1.068 & 0.184 & 0.482 & 1.124 \\
\hline
\end{tabular}

unchanged. Figure 7 shows this trend for $6+8,8+8$ and $8+6$ SL systems under compressive and tensile strain. Table $\mathrm{V}$ summarizes the strained $m \neq n$ cases with their values compared with the corresponding $m=n$ cases. Concluding from all the cases summarized, it is found that both applying tensile strain and decreasing the number of barrier layers weaken the electric fields; therefore, the value of $0.56 \mathrm{MV} /$ $\mathrm{cm}$ in the InN region of a $6+4$ system under tensile strain is the smallest result in this paper.

The VBOs and each component for strained $m=n$ and $m \neq n$ cases are summarized in Table V. It is worth noting that when under compressive (tensile) strain, the interface term of the particular $n+n$ system has the highest (lowest) value among similar geometries, i.e., the corresponding $(n-2)+n$ and $n+(n-2)$ systems. Since the bulk term is independent of the SL geometry where strain is applied, this results in the same trend in VBOs; in other words, the extreme value of VBO belongs to the geometrically intermediate case where strain is present. For instance, regarding systems under compressive strain, the $\mathrm{VBO}$ values of the $8+8$ system $(1.124 \mathrm{eV})$ is higher than that of the $6+8$ $(1.051 \mathrm{eV})$ and $8+6(1.109 \mathrm{eV})$ systems; conversely, where tensile strain is applied, the VBO value of the former system is $0.184 \mathrm{eV}$, which is lower than that of the latter two systems $(0.186 \mathrm{eV}$ and $0.192 \mathrm{eV}$, respectively).

\section{DISCUSSION}

Wei and Zunger ${ }^{14}$ pointed out, as a common expectation, that the bulk term is characterized by the bulk material only, but not the SL structure, while the interface term shows slight dependence on the SL structure. This is expected to be valid for very thick (both well and barrier) SLs. Nevertheless, our results considering $m \neq n$ strain-free systems reveal the relationship between the bulk term and the SL geometry, in that the bulk term can also be engineered by the geometry of the SL, since the geometry affects the resultant (optimized) $a$, which in turn affects the bulk term. Furthermore, both bulk and interface terms decrease as $a$ increases, leading to a negative correlation between the VBO and $a$. Note that the bulk term is the same for all systems under tensile (compressive) strain, which is because where strain is applied, $a$ is fixed and does not depend on the SL geometry, so the bulk term, which is only related to $a$, remains the same. Existing studies of VBOs for the $\mathrm{InN} / \mathrm{GaN}$ heterojunction range widely from $0.48 \mathrm{eV}$ to $1.07 \mathrm{eV}$ (Refs. 3, 10, 13-18), most of which have not considered the dependence on $a$ and the SL geometry, making comparison rather difficult, since our own results vary widely accordingly. As King et al. ${ }^{10}$ mentioned recently, the accuracy of several of these studies are limited by experimental errors or the assumptions made (e.g., neglect of orbitals hybridization). ${ }^{3,15-18}$ Martin et al. ${ }^{13}$ also employed XPS to measure the VBO of the InN/GaN heterojunction, obtaining a VBO value of $1.05 \pm 0.25 \mathrm{eV}$. This result has included a correction term to cancel the piezoelectric effect, based on the false assumption, as pointed out by King et al., ${ }^{10}$ that the piezoelectric polarizations always act to decrease the magnitude of the VBO; it can be seen from our results that according to different strain conditions, piezoelectric polarizations can increase (compressive strain) or decrease (tensile strain) considerably the magnitude of VBOs. Excluding the correction term yields a value of $0.93 \pm 0.25 \mathrm{eV}$ for the $\mathrm{wz} \mathrm{InN} /$ $\mathrm{GaN}(0001)$ heterojunction, which is most likely to result from a sample with an InN film grown coherently on a GaN (a)

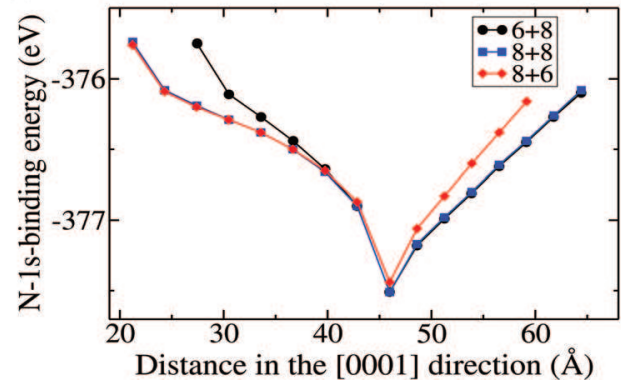

(b)

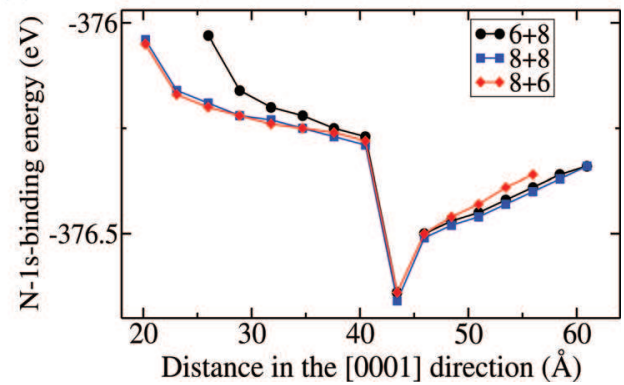

FIG. 7. (Color online) The N-1s binding energy of $6+8,8+8$ and $8+6 \mathrm{wz}$ InN/ $\mathrm{GaN}(0001)$ SLs under (a) compressive strain (in-plane lattice constant fixed as that of GaN "fix-GaN"), and (b) tensile strain ("fix-InN") as a function of $z$, distance in the [0001] direction. The SL systems, $6+4,6+6$ and $6+8$, exhibit similar features. 
TABLE V. The magnitude of the electric fields $|E|$, lattice constants and VBOs of wz InN/GaN(0001) 4+6, $6+4,6+8$ and $8+6$ SLs under tensile, compressive and no strain, compared with the corresponding $m=n$ systems.

\begin{tabular}{|c|c|c|c|c|c|c|c|c|c|}
\hline \multirow{2}{*}{$\begin{array}{l}m \neq n \text { Systems } \\
\text { Relaxation }\end{array}$} & \multicolumn{3}{|c|}{$4+6$} & \multicolumn{3}{|c|}{$6+6$} & \multicolumn{3}{|c|}{$6+4$} \\
\hline & Fix-InN & Free & Fix-GaN & Fix-InN & Free & Fix-GaN & Fix-InN & Free & Fix-GaN \\
\hline$a(\AA)$ & 3.583 & 3.360 & 3.228 & 3.583 & 3.402 & 3.228 & 3.583 & 3.442 & 3.228 \\
\hline$c / n(\AA)$ & 5.321 & 5.516 & 5.624 & 5.405 & 5.574 & 5.723 & 5.489 & 5.627 & 5.821 \\
\hline$|E|_{\mathrm{InN}}(\mathrm{MV} / \mathrm{cm})$ & 1.21 & 6.31 & 9.25 & 0.86 & 3.80 & 5.89 & 0.56 & 2.32 & 5.21 \\
\hline$|E|_{\mathrm{GaN}}(\mathrm{MV} / \mathrm{cm})$ & 1.20 & 5.37 & 8.13 & 1.20 & 5.12 & 8.61 & 1.40 & 4.87 & 11.17 \\
\hline bulk term $(\mathrm{eV})$ & -0.070 & 0.188 & 0.553 & -0.070 & 0.102 & 0.553 & -0.070 & 0.034 & 0.553 \\
\hline interface term $(\mathrm{eV})$ & 0.246 & 0.375 & 0.467 & 0.235 & 0.366 & 0.515 & 0.246 & 0.337 & 0.506 \\
\hline $\mathrm{VBO}(\mathrm{eV})$ & 0.176 & 0.563 & 1.020 & 0.165 & 0.468 & 1.068 & 0.177 & 0.371 & 1.059 \\
\hline$m \neq n$ Systems & & $6+8$ & & & $8+8$ & & & $8+6$ & \\
\hline Relaxation & Fix-InN & Free & Fix-GaN & Fix-InN & Free & Fix-GaN & Fix-InN & Free & Fix-GaN \\
\hline$a(\AA)$ & 3.583 & 3.406 & 3.228 & 3.583 & 3.402 & 3.228 & 3.583 & 3.402 & 3.228 \\
\hline$c / n(\AA)$ & 5.405 & 5.563 & 5.723 & 5.405 & 5.574 & 5.723 & 5.405 & 5.574 & 5.723 \\
\hline$|E|_{\mathrm{InN}}(\mathrm{MV} / \mathrm{cm})$ & 0.93 & 4.99 & 6.32 & 0.71 & 3.41 & 4.18 & 0.59 & 2.51 & 3.99 \\
\hline$|E|_{\mathrm{GaN}}(\mathrm{MV} / \mathrm{cm})$ & 1.08 & 4.92 & 6.84 & 1.13 & 4.63 & 6.87 & 1.40 & 4.67 & 8.52 \\
\hline bulk term $(\mathrm{eV})$ & -0.070 & 0.162 & 0.553 & -0.070 & 0.107 & 0.553 & -0.070 & 0.053 & 0.553 \\
\hline interface term $(\mathrm{eV})$ & 0.255 & 0.384 & 0.497 & 0.254 & 0.375 & 0.571 & 0.262 & 0.354 & 0.556 \\
\hline $\mathrm{VBO}(\mathrm{eV})$ & 0.186 & 0.547 & 1.051 & 0.184 & 0.482 & 1.124 & 0.192 & 0.407 & 1.109 \\
\hline
\end{tabular}

film (compressive strain). This value actually agrees rather well with the lowest value we have within systems under compressive strain ( $1.020 \mathrm{eV}$ for a $4+6$ system).

\section{SUMMARY AND CONCLUSION}

In conclusion, based on extensive DFT calculations, we investigate the built-in electric fields and valance band offsets in $\mathrm{InN} / \mathrm{GaN}(0001)$ SLs, where their correlation with the SL geometry (layer number in both $\mathrm{InN}$ and $\mathrm{GaN}$ regions) and the strain conditions are systematically studied. Spontaneous polarization and the strain-induced piezoelectric polarization are comparable in the contribution to the electric fields. Large built-in electric fields are observed in strainfree wz InN/GaN(0001) SLs, which decrease with increasing number of both well and barrier layers. Furthermore, for strain-free SLs, decreasing only the number of well (barrier) layers results in a stronger (weaker) electric field in the well region, while that in the barrier region barely changes in both cases. Where the strain is applied, the magnitude of the electric fields is strongly affected by the piezoelectric polarizations, suggesting a dominant effect of strain. Tensile strain (fixing the lateral lattice constant to that of $\mathrm{InN}$ ) causes the electric fields in both regions to weaken, while compressive strain (fix-GaN) reinforces them. This is achieved by diminishing the number of well (barrier) layers of strained systems results in stronger electric fields only in the well (barrier) region. The VBO results agree well with several existing studies, with that of the strain-free cases around $0.48 \mathrm{eV}$, and that of the cases under tensile (compressive) strain decreasing (increasing) to $0.165(1.124) \mathrm{eV}$. It is also shown that all bulk, interface terms and the VBOs are dependent on not only the bulk material and the strain conditions, but also the SL geometry (layer numbers of GaN and InN). Our results: the dependence of the built-in electric fields and VBOs on the SL geometry and the strain conditions, represent a hitherto largely unrecognized degree to which these properties can be potentially be controlled, providing more flexibility in device optimization and design.

\section{ACKNOWLEDGMENTS}

We acknowledge the computing resources provided by the National Computational Infrastructure (NCI) Facility. We thank the Australian Research Council for financial support.

${ }^{1}$ J. Wu, J. Appl. Phys. 106, 011101 (2009).

${ }^{2}$ Polarization Effects in Semiconductors, edited by C. Wood and D. Jena (Springer, New York, 2008).

${ }^{3}$ C.-L. Wu, H.-M. Lee, C.-T. Kuo, C.-H. Chen, and S. Gwo, Appl. Phys. Lett. 92, 162106 (2008).

${ }^{4}$ X. Y. Cui, D. J. Carter, M. Fuchs, B. Delley, S. H. Wei, A. J. Freeman, and C. Stampfl, Phys. Rev. B 81, 155301 (2010).

${ }^{5}$ S. F. Chichibu, A. C. Abare, M. P. Mack, M. S. Minsky, T. Deguchi, D. Cohen, P. Kozodoy, S. B. Fleischer, S. Keller, J. S. Speck, J. E. Bowers, E. Hu, U. K. Mishra, L. A. Coldren, S. P. DenBaars, K. Wada, T. Sota, and S. Nakamura, Mater. Sci. Eng., B 59, 298 (1999).

${ }^{6}$ I. Vurgaftman and J. R. Meyer, J. Appl. Phys. 94, 3675 (2003).

${ }^{7}$ U. M. E. Christmas, A. D. Andreev, and D. A. Faux, J. Appl. Phys. 98, 073522 (2005).

${ }^{8}$ C.-L. Wu, C.-H. Shen, and S. Gwo, Appl. Phys. Lett. 88, 032105 (2006).

${ }^{9}$ W. Liang, K. T. Tsen, D. K. Ferry, H. Lu, and W. J. Schaff, Appl. Phys. Lett. 84, 3681 (2004).

${ }^{10}$ P. D. C. King, T. D. Veal, C. E. Kendrick, L. R. Bailey, S. M. Durbin, and C. F. McConville, Phys. Rev. B 78, 033308 (2008).

${ }^{11}$ C. Y. Lai, T. M. Hsu, W.-H. Chang, K.-U. Tseng, C.-M. Lee, C.-C. Chuo, and J.-I. Chyi, J. Appl. Phys. 91, 531 (2002).

${ }^{12}$ N. Grandjean, J. Massies, and M. Leroux, Appl. Phys. Lett. 74, 2361 (1999).

${ }^{13}$ G. Martin, A. Botchkarev, A. Rockett, and H. Morkoc, Appl. Phys. Lett. 68, 2541 (1996).

${ }^{14}$ S.-H. Wei and A. Zunger, Appl. Phys. Lett. 69, 2719 (1996).

${ }^{15}$ C.-L. Wu, H.-M. Lee, C.-T. Kuo, S. Gwo, and C.-H. Hsu, Appl. Phys. Lett. 91, 042112 (2007).

${ }^{16}$ Z. H. Mahmood, A. P. Shah, A. Kadir, M. R. Gokhale, S. Ghosh, A. Bhattacharya, and B. M. Arora, Appl. Phys. Lett. 91, 152108 (2007).

${ }^{17}$ K. Wang, C. Lian, N. Su, and D. Jena, Appl. Phys. Lett. 91, 232117 (2007).

${ }^{18}$ C. F. Shih, N. C. Chen, P. H. Chang, and K. S. Liu, Jpn. J. Appl. Phys. 44, $7892(2005)$ 
${ }^{19}$ Y.-H. Li, A. Walsh, S. Chen, W.-J. Yin, J. Li, J. L. F. Da Silva, X. G. Gong, and S.-H. Wei, Appl. Phys. Lett. 94, 212109 (2009).

${ }^{20}$ P. G. Moses and C. G. Van de Walle, Appl. Phys. Lett. 96, 021908 (2010).

${ }^{21}$ O. Ambacher, J. Majewski, C. Miskys, A. Link, M. Hermann, M. Eickhoff, M. Stutzmann, F. Bernardini, V. Fiorentini, V. Tilak, B. Schaff and L. F. Eastman, J. Phys. Condens. Matter 14, 3399 (2002).

${ }^{22}$ C. G. Van de Walle and J. Neugebauer, Appl. Phys. Lett. 70, 2577 (1997).

${ }^{23}$ J. P. Perdew, K. Burke, and M. Ernzerhof, Phys. Rev. Lett. 77, 3865 (1996).

${ }^{24}$ B. Delley, J. Chem. Phys. 113, 7756 (2000); ibid. 92, 508 (1990).

${ }^{25}$ Strictly speaking, within the supercell approach, there still exists "inherent" strain of the material, even in the fully relaxed superlattices.

${ }^{26}$ S. Massidda, B. I. Min, and A. J. Freeman, Phys. Rev. B 18, R9871 (1987).
${ }^{27}$ S. Picozzi, A. Continenza, and A. J. Freeman, Phys. Rev. B 55, 13080 (1997).

${ }^{28}$ S.-H. Wei and A. Zunger, Appl. Phys. Lett. 72, 2011 (1998).

${ }^{29}$ N. Grandjean, B. Damilano, S. Dalmasso, M. Leroux, M. Laugt and J. Massies, Phys. Status Solidi A 176, 219 (1999).

${ }^{30}$ C. Stampfl and C. G. Van de Walle, Phys. Rev. B 59, 5521 (1999).

${ }^{31}$ A. Zoroddu, F. Bernardini, P. Ruggerone, and V. Fiorentini, Phys. Rev. B 64, 045208 (2001)

${ }^{32}$ W. Paszkowicz, J. Adamczyk, S. Krukowski, M. Leszczynski, and S. Porowski, Philos. Mag. A 79, 1145 (1999).

${ }^{33}$ DAns-Lax Taschenbuch fur Chemiker und Physiker: Band 1, edited by E. Lax and C. Synowietz (Springer, New York, 1967). 